\title{
Potassium shift in thyrotoxic periodic paralysis
}

\author{
JOHN FEELY \\ B.Sc., M.D. \\ Department of Pharmacology and Therapeutics, University of Dundee, Ninewells Hospital and \\ Medical School, Scotland
}

\begin{abstract}
Summary
A thyrotoxic patient who initially presented with periodic paralysis is described. Precipitation of an attack by a high carbohydrate diet was associated with only a modest fall in plasma potassium but with a marked rise in total blood cell potassium.

\section{Introduction}

Periodic paralysis is a rare complication of thyrotoxicosis in British patients there being less than 5 cases reported (Petch, 1964; Dyde, 1965; Papouchado, 1980). The present patient was studied in an attempt to examine the role of insulin in the precipitation of an attack and the possible intracellular influx of potassium associated with paralysis.
\end{abstract}

\section{Case report}

A 30-year-old male night security officer developed repeated attacks of weakness in the legs, being unable to move out of a chair following his rounds, and a weight loss of $25 \mathrm{~kg}$ which led to his dismissal from work. Attacks (2-3/week) were frequently preceded by stiffness in the thigh muscles, the severest during the 10-month course also involved his feet, arms and neck muscles, confining him to bed for $36 \mathrm{hr}$ although he had no difficulty with respiration, speech, eating, bladder or bowel function. A clinical diagnosis of exophthalmic Graves' disease was confirmed by elevated serum thyroxine $425 \mathrm{nmol} / \mathrm{l}$ (normal 75-140 $\mathrm{nmol} / \mathrm{l}$ ) and triiodothyronine $12 \cdot 2$ $\mathrm{nmol} / \mathrm{l}$ (normal 0.9-2.4 nmol/1). Propranolol 160 $\mathrm{mg} /$ day for one week did not influence the attacks; however, a dosage increase to $480 \mathrm{mg} /$ day led to a reduction in the severity and frequency of attacks. To determine the degree of protection by propranolol following an overnight fast and basal samples, the patient commenced a high calorie carbohydrate diet (meals $1200 \mathrm{cal}$ and $400 \mathrm{cal}$ every hour). Venous samples were drawn via an indwelling cannula for measurement of plasma glucose (glucose oxidase method), insulin (radioimmunoassay method), potassium and total blood potassium by flame photo- metry. Total blood cell potassium (TBCK) (normalo 110-180 mmol/l) was calculated from the totalis. blood potassium after correction for packed cell. volume (Lawson et al., 1976). Before the 5.30 p.m. . meal the patient developed paralysis of the thigh muscles, but was able to move his feet. Knee and $\mathrm{C}_{\infty}$ ankle reflexes were brisker than previously and thereo was no loss of sensation.

When euthyroid 3 weeks later, following a partial $\vec{\nabla}$ thyroidectomy and off propranolol, an identical diet including evening meal failed to produce an attack. In the thyrotoxic state, plasma glucose amd $\infty$ insulins were higher throughout and, although.plasma potassium was similar, TBCK was lower except for the marked rise associated with the attack (Fig. 1). No further attacks have occurred.

\section{Discussion}

Periodic paralysis occurs in approximately $2 \%$ of $\overrightarrow{\overrightarrow{0}}$ Chinese and Japanese thyrotoxic patients, usually 3 with Graves' disease (McFadzean and Yeung, 1967). Its rarity among British patients and the $20: 1$ maleo to female ratio has led to speculation concerning therole of genetic predisposition, dietary habits and sex 3 . hormones (Ramsay, 1974). As in the case described, $\dot{\sigma}$ attacks usually occur in the evening following a. large meal or strenuous exercise and are less common $ᄋ$ during cold weather. Electrocardiographic ab-

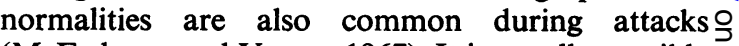
(McFadzean and Yeung, 1967). It is usually possible $\vec{D}$ to demonstrate hypokalaemia during a spontaneous or induced (high carbohydrate intake, insulin or N fludrohydrocortisone) attack, however, in the latter, the fall in plasma potassium may be small 0 (less than $1 \mathrm{mmol} / \mathrm{l}$ ) and within the normal range. $N$ It is now generally accepted (Ramsay, 1974) that the condition is quite distinct from familial periodic 0 paralysis. There is rarely a family history and the $\overparen{\Phi}$ condition occurs in older patients only in association $\stackrel{\mathscr{C}}{+}$ with thyrotoxicosis, although the histological 0 appearance of muscle with vacuolization of sarcomeres and the clinical findings during an attack $\stackrel{\curvearrowright}{\Omega}$ 
are common to both. It has been suggested (Shishiba et al., 1972) that an excessive increase in insulin in response to glucose, changes cell membrane permeability to potassium, and that propranolol may

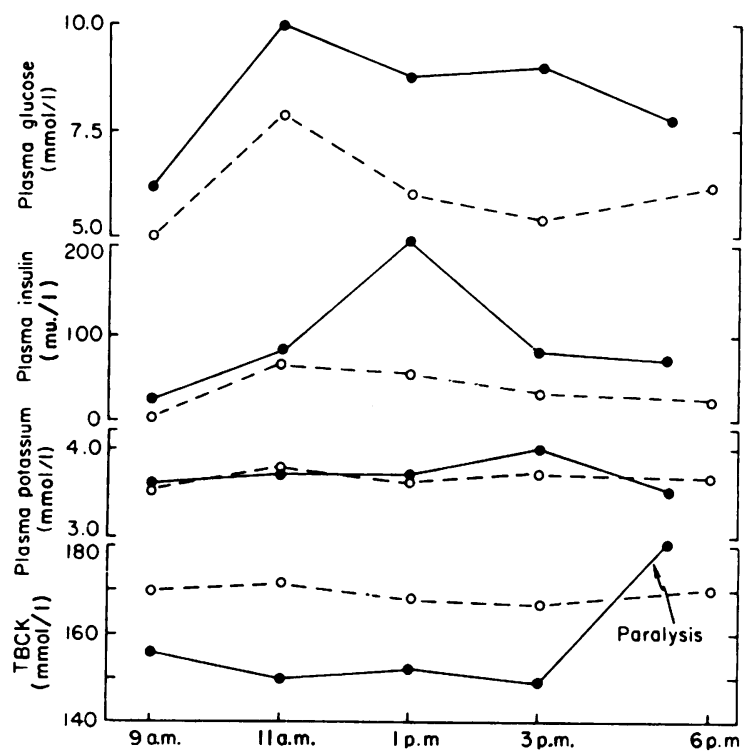

FIG. 1. Laboratory results during a high calorie carbohydrate diet when hyperthyroid ( $-O)$ and when euthyroid ( $O$-. - O). TBCK = total blood cell potassium.

decrease this insulin response. However, in this (Fig. 1) and other cases peak insulin levels occurred well before an attack and Yeung and Tse (1974) found that propranolol did not appear to reduce insulin secretion. Studies on intracellular muscle potassium during paralysis have unexpectedly shown normal or reduced levels (Shishiba et al., 1966), raising the question as to where the potassium goes. The results from this patient suggest a potassium shift into blood cells as one potential site although this clearly requires to be confirmed in additional cases. Also, it does not appear that insulin was responsible for this potassium shift. Propranolol may markedly reduce both the frequency and severity of attacks. As propranolol levels vary widely in thyrotoxic patients and are related to the degree of objective response (Feely et al., 1980), a high dosage, as in this patient, may be required before a satisfactory response is seen. Euthyroidism, however, effects a permanent cure.

\section{Acknowledgments}

I thank Dr S. Venn for TBCK, and Dr T. E. Isles for plasma insulin measurements.

\section{References}

DYDE, J.A. (1965) Hyperthyroidism complicated by periodic paralysis Postgraduate Medical Journal, 41, 286.

Feely, J., Forrest, A., Hamilton, W., GunN, A., Stevenson, I. \& Crooks, J. (1980) Propranolol dosage in thyrotoxicosis. Journal of Clinical Endocrinology and Metabolism, $51,658$.

Lawson, D.H., Boddy, K., Gray, J.M.B., MaHAFFEY, M. \& Mills, E. (1976) Potassium supplements in patients receiving long term oral diuretics for oedema. Quarterly Journal of Medicine (NS), 45, 469.

McFadzean, A.J.S. \& Yeung, R. (1967) Periodic paralysis complicating thyrotoxicosis in Chinese. British Medical Journal, 1, 451.

Papouchado, M. (1980) Thyrotoxic periodic paralysis. Journal of the Royal Society of Medicine, 73, 519.

Petch, C.P. (1964) Thyrotoxic periodic paralysis. (Letter) Lancet, ii, 366.

RAMSAY, I. (1974) Thyroid Disease and Muscle Dysfunction, p. 96. Year Book Medical Publishers, Chicago.

Shishiba, Y., Shimizu, T., Saito, T. \& Shizume, K. (1972) Elevated immunoreactive insulin concentration during spontaneous attacks in thyrotoxic periodic paralysis. Metabolism, 21, 285.

Shishiba, Y., Shizume, K., Sakuma, M., Yamauchi, H., NAKaO, K. \& OKInAKa, S. (1966) Studies on electrolyte metabolism in idiopathic and thyrotoxic paralysis. Metabolism, 15, 153.

YeUNG, R.T.\& TSE, T.F. (1974) Thyrotoxic periodic paralysiseffect of propranolol. American Journal of Medicine, 57, 584. 